\title{
Performance Evaluation for a Unicast Non Delay Tolerant Position based Routing Protocols in VANETs
}

\author{
Ahmed Mohamed Abdalla \\ College of Technological Study \\ PAAET, Kuwait
}

\begin{abstract}
Vehicular Ad hoc Networks are special kind of Mobile Ad Hoc Networks. VANET is an emerging technology, which enables an extensive range of applications, including road safety, passenger convenience, self-driven vehicles, and intelligent transportation.
\end{abstract}

Routing in Vehicular Ad hoc Networks is a challenging task due to the unique characteristics of the network. Unicast routing protocols in VANETs are the most fundamental protocols in ad hoc environment and they form the basis for constructing other types of protocols. Unicast routing protocols further classified into topology based, position based, cluster based and hybrid protocols.

In this paper protocols belonging to unicast non-delay tolerant position based are discussed. We have implemented our comparison on the NS2 simulator. Simulation of NDT routing protocols A-STAR, CAR, and GyTAR are carried out and the results are presented.

\section{Keywords}

Routing Protocols, VANETs, Position based routing.

\section{INTRODUCTION}

Vehicular Ad hoc Networks (VANETs) are special kind of Mobile Ad Hoc Networks (MANETs) that are formed between moving vehicles and/or the nearest Road Side Units (RSUs). VANET is an emerging technology, which enables an extensive range of applications, including road safety, passenger convenience, self-driven vehicles, and intelligent transportation [1].

Wireless communication among moving vehicles is increasingly the focus of research in our modern life. Automobile industry is now driven by the requirement of selfdriven vehicles that need the exchange of information among vehicles to improve the safety, and comfort of drivers and passengers [2-4].

Changing in rapid topology and frequent disconnection paths makes it difficult to design an efficient routing protocol for routing data among vehicles. There are two types of communications in VANETs Vehicle to Vehicle (V2V) communication and Vehicle to road side Infrastructure (V2I).

Routing in Vehicular Ad hoc Networks is a challenging task due to the unique characteristics of the network such as high mobility of vehicles, dynamically changing topology and highly partitioned network. It is a challenge to ensure reliable, continuous and seamless communication route in the presence of moving vehicles.

In unicast routing protocols data packets are transmitted from a single source to a single destination. Unicast routing protocols are the most fundamental protocols in ad hoc networks and they form the basis for constructing other types of protocols. Unicast routing protocols are supplementary classified into topology based, position based, cluster based and hybrid protocols [4].

In position-based routing protocols, all vehicles recognize their own locations and their neighbor vehicle geographic locations through position-pointing devices such as Global Position System (GPS). A GPS device does not made any routing table or exchange any information related to the link state with the neighbor vehicles. The information from the GPS device is used in making routing decisions. This type of routing performs better as creating and maintaining a total route path from the source vehicle to the destination vehicle are not necessary. The position-based routing protocols can be categorized as nondelay tolerant network (non-DTN) routing protocols, delay tolerant network (DTN) routing protocols, and hybrid routing protocols.

This paper focuses on unicast non-delay tolerant position based routing protocols in VANET environment and presents a detailed review of A-STAR, CAR and GyTAR routing protocols.

The rest of the paper is structured as follows. Vehicular Ad hoc Networks illustrated in section 2. Section 3 discusses the issues of routing in VANETs. Section 4 presents VANETs unicast position based routing protocols. Section 5 presents the simulation results and finally Section 6 discussion.

\section{VEHICULAR AD HOC NETWORKS}

Vehicular Ad hoc Networks (VANETs) are considered subclass of Mobile Ad Hoc Networks (MANETs) that are formed between moving vehicles and/or the nearest fixed Road Side Units (RSUs). VANET is an emerging technology, which enables an extensive range of uses, including road safety, passenger convenience, self-driven vehicles, and intelligent transportation $[1,2]$

Efficient routing protocols help to make roads safer by spreading information about the road conditions and traffic scenario among the contributing vehicles within a very short period of time.

VANETs enable also automated both city and highway applications, where the vehicles are able to voyage without the help of their drivers; such applications have yet become realistic and demanded. VANETs possess certain unique characteristics such as high mobility of vehicles, time varying density of vehicles, highly divided network, repeated disconnections and dynamically changing for networks topology, which makes them more challenging [5]. It is a challenge to build robust networks between vehicles and ensure continuous, secure, and reliable communication paths among the neighbor vehicles in motion [4].

The federal communications commission (FCC) has allocated $75 \mathrm{MHz}$ of spectrum in the $5.9 \mathrm{GHz}$ band for vehicle-vehicle and vehicle-roadside communication, called dedicated short 
range communications (DSRC). IEEE is also working on the IEEE 1609 family of standards for wireless access in vehicular environments (WAVE), which define architecture and a complementary, standardized set of services and interfaces that collectively enable secure vehicle-to vehicle (V2V), vehicle to infrastructure (V2I) and vehicle-to- road side (V2R) wireless communications. Due to rapid of networks topology changing and frequent disconnection makes it difficult to design an efficient routing protocol for routing data among vehicles [6].

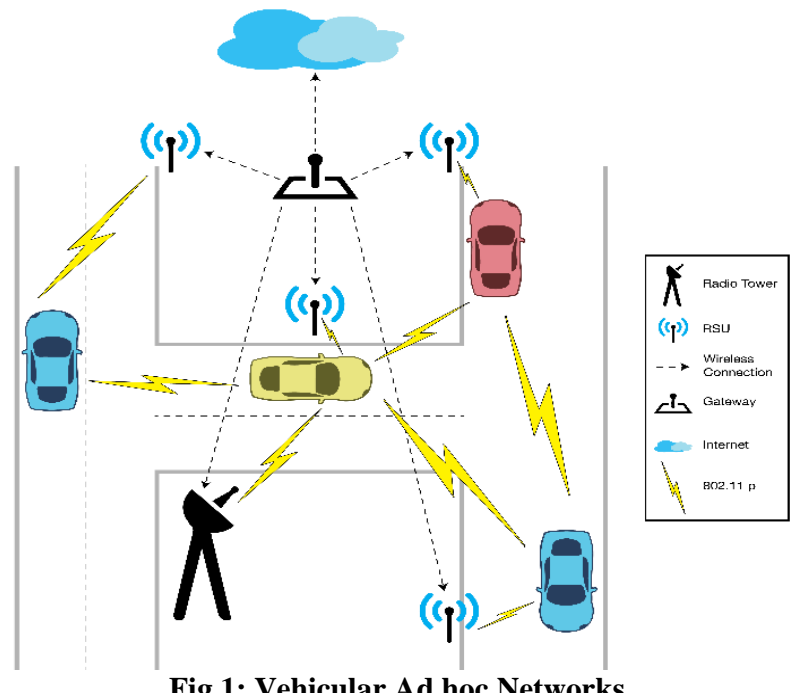

Fig 1: Vehicular Ad hoc Networks

\section{VANETS ROUTING PROTOCOLS}

Reliable and fast routing in VANETs are the challenging tasks due to the unique characteristics of the network such as high mobility of vehicles, dynamically changing topology and highly partitioned network. It is a challenge to ensure reliable, continuous and seamless communication in the presence of fastmoving vehicles. The performance of VANETs routing protocols depends on many internal factors such as mobility of vehicles and external factors such as road topology. This demands a highly adaptive approach to deal with the dynamic circumstances by selecting the best routing and forwarding strategies and by using appropriate transmission and mobility models.

\subsection{Unicast Routing Protocols}

Unicast routing protocols only transmit data packets from a single source to a single destination. They are primarily required to support personalized applications and commercial applications such as internet connectivity and multimedia access.

\subsection{Broadcast Routing Protocols}

This is the most common routing protocol used in VANETs, especially in safety related applications. Flooding is a noticeable technique used in broadcast routing protocols. However, blind flooding leads to broadcast storm problem. Broadcast storms make channel capacity to be overloaded, causing channel congestion that reduces communication reliability. Broadcast protocol is suitable only for small number of vehicles in the network [7-8].

\subsection{Multicast/Geocast Routing Protocols}

Multicast Routing enables spreading of messages from single source to a group of destination vehicles of interest. Geocast routing is basically a location based multicast routing, which aims to deliver information from a source vehicle to all other vehicles within a specified geographical region called a Zone of

\section{Relevance (ZOR) [9].}

\subsection{Cluster Based Routing Protocols}

Clustering in vehicular ad hoc network can be defined as the virtual partitioning of the dynamic vehicles into several groups. A group of vehicles identify themselves to be part of a cluster. A special vehicle, selected as cluster-head is responsible for routing, spreading of inter cluster traffic, scheduling of intracluster traffic and channel assignment for cluster members. The cluster members do not contribute in routing [4].

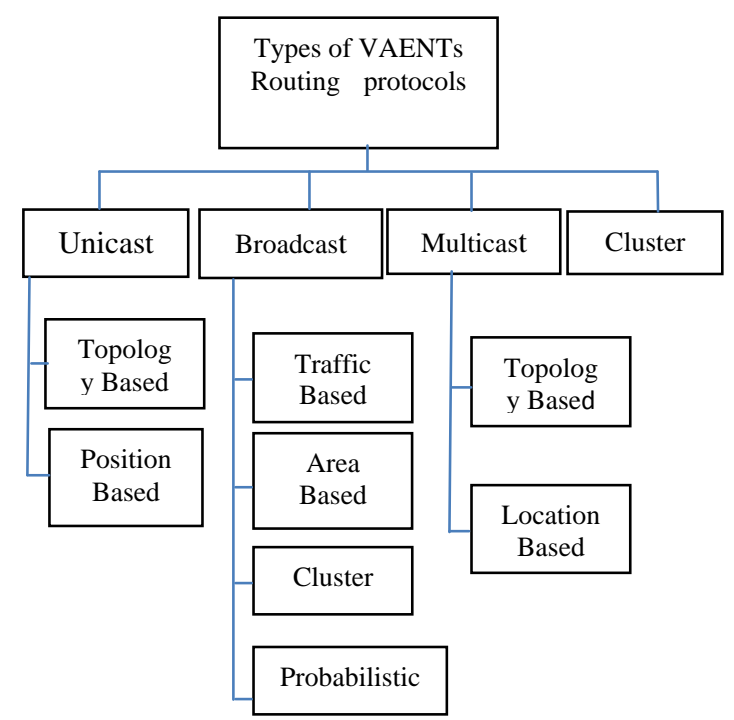

Fig 2: Types of VANETs Routing Protocols.

\section{UNICAST POSITION-BASED ROUTING PROTOCOLS}

Unicast routing protocols are the most fundamental protocols in ad hoc environment and they form the basis for constructing other types of protocols. Unicast routing protocols are categorized into topology based, position based, cluster based and hybrid protocols [4].

Topology based routing protocols do not efficient in VANETs due to the overheads relating to the discovery of routes and maintenance of routes in the presence of moving vehicles. In VANET environment the mobility factor is very high, which leads to the frequent network partitioning and route disconnection demanding re-computation propagation paths according to a new topology information.

In position based protocols, the routing decisions are based on geographic position of the vehicles. This does not require establishment or maintenance of routes, but needs location services to determine the position of the destination. Some of the commonly used location services include Simple Location Services (SLS), Reactive Location Services (RLS), DREAM Location Services (DLS) and Global Position System (GPS) [4]. In position based protocols, the packet is sent without any knowledge of digital map to the one-hop neighbor, which is the closest to the position of the destination vehicle. Every vehicle must continuously send beacon (Hello) packets with their position information and other vehicle identification parameters. Position based protocols are suitable for VANETs since they offer higher delivery ratio than topology based routing protocols in a highly mobile environment [10]. They provide minimum delay in establishing the route path and achieve good scalability. 
Forwarding mechanisms have been used by position based routing protocols consisted of many types like greedy forwarding, trajectory based forwarding, opportunistic forwarding, contention based forwarding and hybrid forwarding. Position based approaches are further classified into non-delay tolerant network, delay tolerant network, and hybrid.

In position-based routing protocols, all vehicles recognize their own locations and their neighbor vehicle geographic locations through position-pointing devices such as GPS. It does not manage any routing table or exchange any information related to the link state with the neighbor vehicles. The information from the GPS device is used in making routing decisions. This type of routing performs better as creating and maintaining a global route from the source vehicle to the destination vehicle are not necessary. The position-based routing protocols can be classified as non-delay tolerant network (non-DTN) routing protocols, delay tolerant network (DTN) [11] routing protocols, and hybrid routing protocols. Classification of position based routing protocols is illustrated in figure 3 . In this paper we are focused on non-DTN routing protocols.

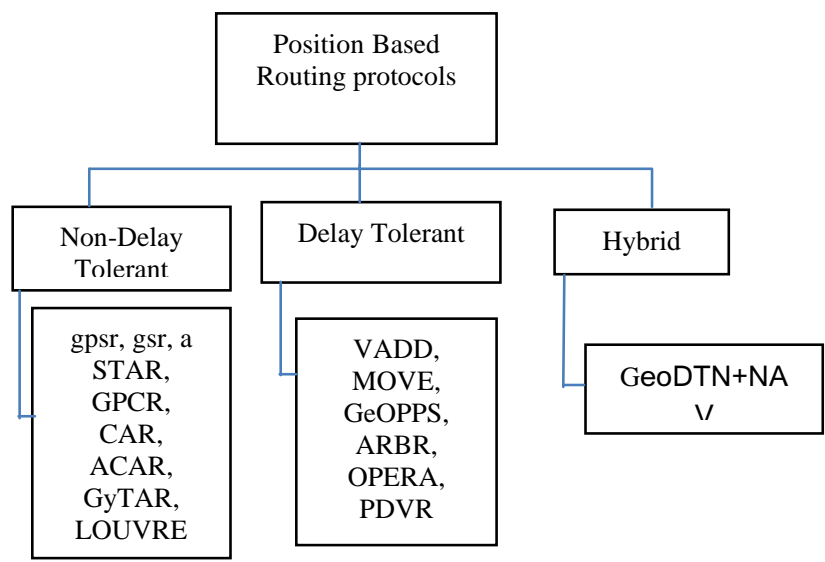

Fig 3: Types of Position Based Routing Protocols.

\section{Non-Delay Tolerant Network (Non-DTN)}

Non-delay tolerant network protocols are also identified as minimum delay protocols and they goal at minimizing the delivery time of the information from source node to destination node. Non-DTN protocols are appropriate for real time safety applications, which request a critical time response during data distribution [4]. Delaying time in the transmission is the main parameter in the design of Non-DTN protocols and the shortest path method is usually implemented. The shortest path method may not always guarantee faster delivery, especially when the traffic condition is light [5].

Greedy forwarding technique is the commonly used in VANETs, where the packet is forwarded to a neighbor node which is geographically closer to the destination node. If a packet reaches a node which has no neighbors that is closer to the destination, a local maxima problem occurs. Each routing protocol in this group applies its own recovery strategy to overcome the local maxima problem. One of the earliest protocols used in Greedy protocols is Greedy Perimeter Stateless Routing (GPSR). GPSR makes greedy forwarding decisions only by using information about the instant neighbors of the routing vehicle in the network. In case of local maxima occurs, GPSR used perimeter forwarding technique for recovery. GPSR uses many models for mobility prediction, which considers the mobility of sets of vehicles [4].

GPSR routing protocol works well in highway environment but achieve low performance in city environment. GPSR relies on distributed algorithm for graphs. In the presence of radio problems such as tall buildings in city environment, these algorithms frequently partition an otherwise connected graph, making the delivery of packets impossible [10].

Geographic Source Routing (GSR) merging position based routing with topological knowledge. GSR gets the position information of the destination node by using RLS system and uses a city map for complete information of city topology [4]. Greedy Perimeter Coordinator Routing (GPCR) also takes advantage of the fact that city streets form a natural planner graph. GPCR improves GSR by rejecting the use of external street map. The protocol consists of two parts: a restricted greedy forwarding procedure, which forwards the messages to the vehicles at junction and a repair strategy, which is based on the topology of real world streets and junctions [11].

Anchor based Street and Traffic Aware Routing protocol (ASTAR) is another position based routing protocol, A-STAR routing protocol is designed specifically for inter-vehicular communication systems in city environment. A-STAR is used data collected from city bus routes to identify an anchor path from source to destination with high connectivity for packet delivery [12]. A-STAR routing protocol uses a more efficient recovery strategy for local maxima problem. It computes new anchor paths for recovery and also announces the void area causing local maxima problem temporarily unreachable. It provides better performance as compared to both GSR and GPCR routing protocols.

Connectivity-Aware Routing protocol (CAR) has distinctive features that enable it to maintain the cache of optimal route paths between numerous source and destination pairs. Prediction the position of destination vehicle reformations route can be done by CAR routing protocol in case there is a change in position.

Greedy Traffic Aware Routing protocol (GyTAR) goals is to achieve efficient use of network bandwidth and aims at achieving reduced end-to-end delay and packet loss. It adopts a new intersection based geographical routing protocol capable of finding robust routes in city environment with multi lanes [15].

These protocols aim to transmit data packets to the destination as soon as possible. The basic outlook in the greedy approach of non-DTN routing protocols is that a vehicle advances its packet to its neighbor, which is close to the destination. However, the forwarding strategy might be unsuccessful if the neighbors are not nearer to the destination than the vehicle. The routing protocols in this group have their individual recovery approach to overcome such failures.

This research focuses on unicast non-delay tolerant position based routing protocols in VANET environment and presents a detailed review of A-STAR, CAR and GyTAR routing protocols.

\section{Anchor based Street and Traffic Aware Routing (A-STAR) Routing Protocol}

A-STAR is a position-based routing scheme [12], A-STAR protocol is designed especially for Inter Vehicle Communication System (IVCS) in a city environment.

Unique to A-STAR is that it used information collected from city bus routes to compute an anchor path from source vehicle to destination vehicle with high connectivity for packet delivery. A-STAR also used a new recovery strategy for packets routed to a local maximum.

A-STAR routing protocol used street map to calculate the chain of junctions (anchors) through which a packet has to propagate 
to reach to its destination node. Integration of traffic awareness is used by A-STAR routing protocol by employing statistically evaluated maps (determine the anchor paths of optimum connectivity by keeping track of the number of city bus routes on each street) or dynamically evaluated maps (dynamically inspecting the state of the latest traffic to recognize the best anchor paths) to determine an anchor path with a high connectivity for packet delivery. A-STAR utilizes a novel local recovery strategy for packets routed to a local minimum, which is more appropriate for urban environment, as against the greedy approach of GSR and the recovery mode of GPSR. ASTAR overcomes the short comings of GSR and provides a new recovery strategy which computes an anchor path from the local maximum through which the packet is routed [11]. The disadvantage of A-STAR is that it uses static information based on city bus routes which causes connectivity problem on some portion of streets due to no bus route.

Table 1. Advantages and Disadvantages of A-STAR [13]

\begin{tabular}{||l|l||}
\hline \multicolumn{1}{|c|}{ Advantages } & \multicolumn{1}{|c|}{ Disadvantages } \\
\hline $\begin{array}{l}\text { Path selection of A-STAR } \\
\text { ensures high connectivity } \\
\text { though its packet delivery } \\
\text { ratio is lower than GSR \& } \\
\text { GPSR. }\end{array}$ & $\begin{array}{l}\text { Packet delivery ratio of A- } \\
\text { STAR is low. }\end{array}$ \\
\hline $\begin{array}{l}\text { A-STAR uses a new local } \\
\text { recovery strategy which is } \\
\text { more suitable for city } \\
\text { environment. }\end{array}$ & $\begin{array}{l}\text { To find a path from source to } \\
\text { destination it uses static } \\
\text { information based on city } \\
\text { connectivity problem on on } \\
\text { some portion of streets due } \\
\text { to no bus route there. }\end{array}$ \\
\hline $\begin{array}{l}\text { In low traffic density, A- } \\
\text { STAR ensures for finding an } \\
\text { end-to-end connection. }\end{array}$ & \multicolumn{2}{|l}{} \\
\hline
\end{tabular}

\section{Connectivity-Aware Routing (CAR)}

Connectivity-Aware Routing protocol (CAR) [14] designed especially for inter-vehicle communication in a metropolitan and/or highway environment. CAR protocol integrates destinations location with finding connected paths between source vehicle and destination vehicle. Once a path is created, path is automatically adjusted on the fly to account for changes, without needs to another discovery process. "Guards" help to track the current position of a destination node, even if the destination travels a substantial distance from its initially known location.

CAR protocol has typical features that enable it to maintain the cache of effective route between numerous source and destination pairs.

Prediction the position of destination vehicle reformations route can be done by CAR routing protocol in case there is a change in position. The vehicles that used CAR protocol transmit periodical Hello beacons which comprise their velocity vector data. A vehicle will register the sender in its neighbor table and compute its velocity as well as the velocity of its neighbor as soon he that vehicle receives the Hello beacons. To reduce the loss of bandwidth and congestion, beacons might be piggybacked as well on proceeded data packets. In case of accesses will be unreachable from the neighbor table because the space between the vehicles exceeds the threshold rate. The CAR protocols setup a guard message which is a geographical marker message; it is buffered and transmitted from one vehicle to another to propagate the new information. Two models of guards can be presented by CAR which are called the traveling guard and the standing guard. Routing errors may either result from a gap in the communication between the anchor points or due to problems in the guards themselves. To overcome that CAR protocol devised two recovery strategies. The first called the time out algorithm with active waiting cycle and the second is called the walk around error recovery. The CAR protocol has a unique merit that is not found in other protocols which that it is able to create virtual information in the form of guards [14]. The main disadvantage of CAR are that unnecessary nodes can be selected as an anchor and when the traffic environment changes CAR protocol cannot adjust him with different subpath

Table 2. Advantages and Disadvantages of CAR [13].

\begin{tabular}{|l|l||}
\hline \multicolumn{1}{|c|}{ Advantages } & \multicolumn{1}{|c|}{ Disadvantages } \\
\hline $\begin{array}{l}\text { CAR ensures to find the } \\
\text { shortest connected path. }\end{array}$ & $\begin{array}{l}\text { Unnecessary nodes can be } \\
\text { selected as an anchor. }\end{array}$ \\
\hline $\begin{array}{l}\text { It has no local maximum } \\
\text { problem. }\end{array}$ & $\begin{array}{l}\text { It cannot adjust with different } \\
\text { sub-path when traffic } \\
\text { environment changes. }\end{array}$ \\
\hline No digital map is required & \\
\hline
\end{tabular}

Greedy Traffic Aware Routing protocol (GyTAR)

Greedy Traffic Aware Routing protocol [15] gives a new concept of intersection-based routing protocol. Greedy Traffic Aware Routing protocol goals is to achieve efficient use of network bandwidth and aims at achieving reduced end-to-end delay and packet loss. GyTAR implements a new intersection based geographical routing protocol capable of finding robust routes paths in city environment with multi-lanes. GyTAR is an overlaid approach packets are forwarded toward the next junction which will then determine the best junction to forward next. GyTAR assumes that the roadside units will send the number of vehicles per each road and also determines the connectivity of roads.

The key features of the GyTAR protocol are the effectively selection of the junctions to find possible routes and employs a carry-and-forward approach to recover from the local maximum [16].

GyTAR also utilizes a digital map to recognize the location of neighboring junctions and efficiently selects the connection based on the traffic density and curve-metric distance to the destination. A score is given to all neighboring junctions based on the traffic density and the curve-metric distance to the destination [16].

The main disadvantage of GyTAR protocol is that it depends on roadside units because it assumes that the number of cars in the road will be given from road side units.

Table 3. Advantages and Disadvantages of GyTAR [13]

\begin{tabular}{|l|l||}
\hline \multicolumn{1}{|c||}{ Advantages } & \multicolumn{1}{c||}{ Disadvantages } \\
\hline $\begin{array}{l}\text { For high mobility topology } \\
\text { changes rapidly and often } \\
\text { occurring network } \\
\text { fragmentation which is } \\
\text { efficiently handle by } \\
\text { GyTAR. }\end{array}$ & $\begin{array}{l}\text { GyTAR depends on } \\
\text { roadside units because it } \\
\text { assumes that the number of } \\
\text { cars in the road will be } \\
\text { given from road side units. }\end{array}$ \\
\hline $\begin{array}{l}\text { Performance shows that } \\
\text { throughput, delay and } \\
\text { routing overhead are better } \\
\text { than GSR. }\end{array}$ & Gytar cannot avoid void. \\
\hline
\end{tabular}




\section{SIMULATION RESULTS AND DISCUSSIONS}

Table 4. Simulation Parameters Simulation parameters

\begin{tabular}{|l|l|}
\hline Network simulator & NS-2 version 2.34 \\
\hline Simulation Time & 300 seconds \\
\hline Map Size & $2000 \mathrm{~m}$ x $1500 \mathrm{~m}$ \\
\hline Mobility Model & M-Grid \\
\hline Vehicles speed & $20,40,60,80 \mathrm{~km}$ \\
\hline Number of vehicles & $80-180$ \\
\hline MAC protocol & $802.11 \mathrm{DCF}$ \\
\hline Channel Capacity & $2 \mathrm{Mbps}$ \\
\hline Trans. Range & $250 \mathrm{~m}$ \\
\hline Traffic Model & $15 \mathrm{CBR}$ connections \\
\hline Packet sending rate & 4 packets / second \\
\hline Data packet size & 128 bytes \\
\hline Channel type & Wireless channel \\
\hline Antenna Model & Omni directional \\
\hline
\end{tabular}

We have considered the routing protocols A-STAR, CAR, and GyTAR which are unicast NDT position based protocols. Figure 4 exhibits the node density versus the packet delivery ratio. It is quite evident from the graph that GyTAR protocol is performing much better than A-STAR and CAR routing protocols. A-STAR routing protocol packet delivery ratio was low compared to the other protocols although the increased in node density. That due to A-STAR is depends on the information from a city buses that causes connectivity problem on some portion of routing path due to no bus route there. Also, from the graph it is clear that in general the packet delivery ratio is improved as the node density increased. This is due to the fact that as the node density increased the number of trusted routing paths from the source to the destination was increased.

Figure 5 show the nodes density versus the End-to-End delay. It is obvious from figure 5 that the performance of CAR routing protocol is inferior compared to A-STAR and GyTAR routing protocols especially when the nodes density are low. The results indicate that CAR routing protocol performance is improved as the number of nodes density is increased. That is due to that CAR ensures to find the shortest connected path. Figure 5 also illustrate that GyTAR routing protocol achieve lower End-toEnd delay compared to A-STAR and CAR regardless of the number of nodes density.

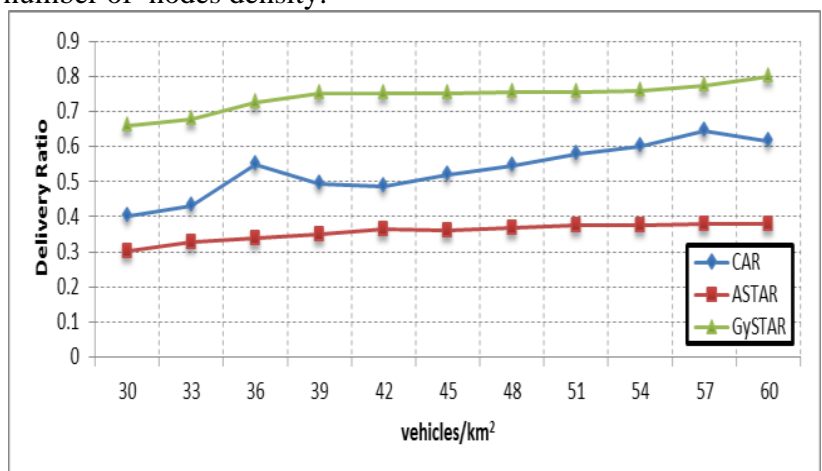

Fig 4: Node Density Vs Delivery Ratio.

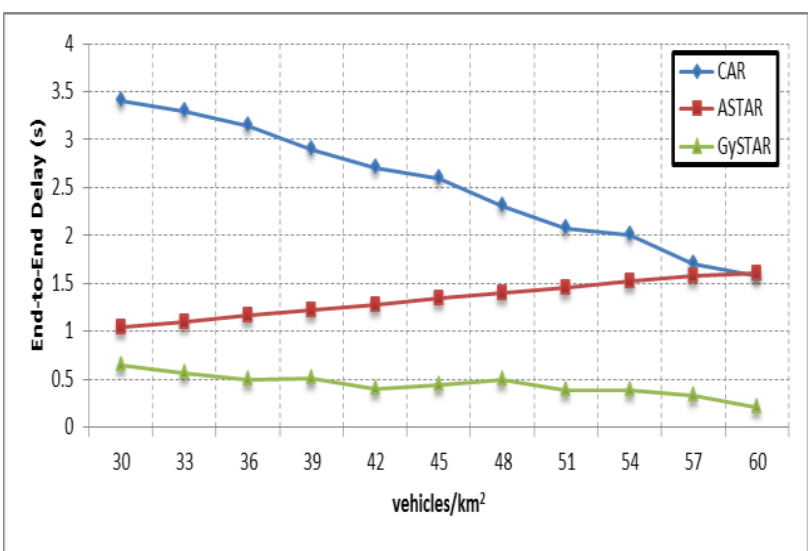

Fig 5: Node Density Vs End-to-End Delay.

\section{DISCUSSIONS}

Performance of routing protocol for VANETs depends on many factors such as the mobility of vehicular, vehicular density, rapid topological changes and other driving environment. It also depends on the use of appropriate mobility model and propagation model. VANETs protocol should perform well in both dense and light traffic conditions either in city or highways seamlessly. In this paper protocols belonging to unicast nondelay tolerant position based are discussed. We have implemented our comparison on the NS2 simulator. Simulation of NDT routing protocols A-STAR, CAR, and GyTAR are carried out and the results are presented. According to simulation results GyTAR routing protocol is achieved better performance than A-STAR and CAR routing protocols.

In the next research paper we will try to discuss delay tolerant network (DTN) routing protocols to determine the performance difference with Non-DTN routing protocols.

\section{REFERENCES}

[1] Venkatesh, Indra. A and Murali. R, "Vehicular Ad hoc Networks (VANETs): Issues and Applications", Journal of Analysis and computation, Vol. 8, No. 1, 2012, pp.31-46.

[2] Sardar Muhammad Bilal, Carlos Jesus Bernardos, Carmen Guerrero, Position-based routing in vehicular networks: "A survey, In Journal of Network and Computer Applications", Volume 36, Issue 2, 2013, Pages 685-697, ISSN 1084-8045,

[3] Baraa T. Sharef, Raed A. Alsaqour, Mahamod Ismail, "Vehicular communication ad hoc routing protocols: A survey", In Journal of Network and Computer Applications, Volume 40, 2014, Pages 363-396, ISSN 1084-8045, https://doi.org/10.1016/j.jnca.2013.09.008.

[4] Venkatesh, A Indra, R Murali, "Routing Protocols for Vehicular Adhoc Networks (VANETs): A Review", Journal of Emerging Trends in Computing and Information Sciences, Vol. 5, No. 1, pp. 25-43, January 2014 ISSN 2079-8407.

[5] Felipe Cunha, Leandro Villas, Azzedine Boukerche, Guilherme Maia, Aline Viana, Raquel A. F. Mini, Antonio A. F. Loureiro, "Data communication in VANETs: Protocols, applications and challenges", In Ad Hoc Networks, Volume 44, 2016, Pages 90-103, ISSN 15708705 ,

[6] Yang, Q., Lim, A., Li, S., Fang, J., \& Agrawal, P. (2010). ACAR: Adaptive connectivity aware routing for vehicular ad hoc networks in city scenarios. Mobile Networks and 
Applications, 15(1),

$36-60$.

doi:http://dx.doi.org/10.1007/s11036-009-0169-2

[7] Saif Al-Sultan, Moath M. Al-Doori, Ali H. Al-Bayatti, Hussien Zedan, "A comprehensive survey on vehicular Ad Hoc network", In Journal of Network and Computer Applications, Volume 37, 2014, Pages 380-392, ISSN 1084-8045,

[8] Xian Shi, Gang Zhang, Chunfeng Liu, Tingting Han, "A Novel Trace-Aided Data Dissemination Algorithm in Vehicular Networks", Ubiquitous Intelligence and Computing and 2015 IEEE 12th Intl Conf on Autonomic and Trusted Computing and 2015 IEEE 15th Intl Conf on Scalable Computing and Communications and Its Associated Workshops (UIC-ATC-ScalCom) 2015 IEEE 12th Intl Conf on, pp. 99-104, 2015.

[9] Dan Lin, Jian Kang, Anna Squicciarini, Yingjie Wu, Sashi Gurung, Ozan Tonguz, "MoZo: A Moving Zone Based Routing Protocol Using Pure V2V Communication in VANETs", Mobile Computing IEEE Transactions on, vol. 16, pp. 1357-1370, 2017, ISSN 1536-1233.

[10] Tesnim Mekki, Issam Jabri, Abderrezak Rachedi, Maher ben Jemaa, "Vehicular cloud networks: Challenges, architectures, and future directions", In Vehicular Communications, Volume 9, 2017, Pages 268-280, ISSN 2214-2096,

[11] S. Jain, K. Fall, and R. Patra, "Routing in a Delay Tolerant
Network", in ACM SIGCOM'04, Portland, Oregon, USA, August, 2004.

[12] Seet, Boon-Chong \& Liu, Genping \& Lee, Bu \& Foh, Chuan \& Juan Wong, Kai \& Lee, Keok-Kee. (2004). "ASTAR: A Mobile Ad Hoc Routing Strategy for Metropolis Vehicular Communications". Proc. IFIP TC6 Networking. 3042. 989-999. 10.1007/978-3-540-24693-0_81.

[13] Bijan Paul, Md. Ibrahim and Md. Abu Naser Bikas. Article: VANET Routing Protocols: Pros and Cons. International Journal of Computer Applications 20(3):28-34, April 2011.

[14] V. Naumov and T. R. Gross, "Connectivity-Aware Routing (CAR) in Vehicular Ad-hoc Networks," IEEE INFOCOM 2007 - 26th IEEE International Conference on Computer Communications, Anchorage, AK, 2007, pp. 1919-1927. doi: 10.1109/INFCOM.2007.223

[15]Jerbi, M \& Senouci, S.-M \& Meraihi, Rabah \& GhamriDoudane, Yacine. (2007). "An Improved Vehicular Ad Hoc Routing Protocol for City Environments". IEEE International Conference on Communications. 3972 3979. 10.1109/ICC.2007.654.

[16] S. M. Bilal, S. Mustafa and U. Saeed, "Impact of directional density on GyTAR routing protocol for VANETs in city environments," 2011 IEEE 14th International Multitopic Conference, Karachi, 2011, pp. 296-300. 